\title{
COX-2, p16 and Ki67 expression in DCIS, microinvasive and early invasive breast carcinoma with extensive intraductal component
}

\author{
Bartova $\mathrm{M}^{1}$, Ondrias $\mathrm{F}^{2}$, Muy-Kheng $\mathrm{T}^{3}$, Kastner $\mathrm{M}^{3}$, Singer $\mathrm{Ch}^{3}$, Pohlodek $\mathrm{K}^{1}$ \\ 2nd Department of Obstetrics and Gynaecology, Comenius University, School of Medicine, University Hospital of Bratislava, \\ Slovakia.kpohlodek@gmail.com
}

\begin{abstract}
Background: Recent studies have showed a significant association between the combination of COX-2, p16 and Ki67 overexpression and incidence of subsequent invasive carcinoma in a subgroup of treated ductal carcinoma in situ (DCIS) and the indicated prognostic value of COX-2, p16 and Ki67 in early breast cancer. Based on the continual model of carcinogenesis and the mentioned results, we hypothesize, that if COX-2, p16 and Ki67 expression is prognostic for DCIS future behaviour, the expression level of the markers correlates also with different stages of breast carcinomas such as DCIS, microinvasive cancer and early invasive cancer with an extensive intraductal compound. The aim of this study was to compare the expression of COX-2, p16 and Ki67 in different stages of breast carcinoma such as pure DCIS, microinvasive cancer (T1mic) and invasive ductal carcinoma with an extensive intraductal component (IDC with EIC). The expression was assessed only in in situ component of the three subgroups (DCIS, T1mic, EIC) in order to show a possible correlation of COX-2, p16 and Ki67 with different stages of carcinogenesis. Methods: We carried out a retrospective study using immunohistochemical staining to evaluate the expression of the markers COX-2, p16 and Ki67 in in situ lesions within three subgroups of tumors with the rising extant of invasive compound: in pure DCIS, microinvasive carcinoma (T1mic) and invasive carcinoma with extensive in situ component (IDC with EIC). Additionally, we performed a correlation analysis between the tumor subgroups and patients history data (age, parity, age of menarche, family and personal cancer history, breast feeding lengths, contraception intake, chest irradiation) as well as some of the tumor characteristics (tumor grade, multicentricity, necrosis). Results: Distribution of p16 expression differed significantly among the three diagnoses. P16 score 1 was highest in the DCIS group whereas the lowest proportion was in IDC and p16 overexpression (score 2,3$)$ maintained this tendency (overexpression proportion in DCIS $<$ T1mic $<$ IDC), though this was not significant. The frequency of COX2 and p16 overexpression (phenotype COX-2+p16+) was higher in EIC within invasive carcinoma in comparison to DCIS and T1mic and was rising gradually with the severity of the diagnosis (proportion in DCIS $<$ T1mic $<$ IDC). Conclusion: This is the first published study ever assessing the expression of COX-2, p16 and Ki67 markers in different breast tumors containing DCIS compound. Our results showed an increasing expression pattern of COX-2 and p16 with the rising severity of the diagnosis (expression was measured exclusively in in situ lesions within tumors containing different extant of invasiveness). The same relationship was showed for p16 marker alone. These data support different expression pattern of COX-2 and p16 markers in combination and p16 marker alone in "in situ lesions" according to the stage of carcinogenesis. This fact might be useful in the evaluation of further behaviour of early breast tumors (Tab. 3, Fig. 8, Ref. 29). Text in PDF www.elis.sk. Key words: COX-2, p16, Ki67 expression, prognosis, biomarkers, early breast cancer.
\end{abstract}

Ductal carcinoma in situ of the breast (DCIS) is known as early form of breast cancer and non-obligatory precursor of invasive cancer. Incidence of DCIS has dramatically increased with routine mammographic screening. Regarding this fact, a question of optimal treatment of DCIS has come to foreground in the field of breast cancer management.

2nd Department of Obstetrics and Gynaecology, Comenius University School of Medicine, University Hospital of Bratislava, Slovakia, ${ }^{2}$ Alpha medical patológia s.r.o., University Hospital Bratislava, Slovakia, and ${ }^{3}$ Department of Obstetrics and Gynaecology, Medical University of Vienna, Vienna General Hospital, Austria

Address for correspondence: K. Pohlodek, MD, PhD, 2nd Department of Obstetrics and Gynaecology, University Hospital of Bratislava, Ruzinovska 6, SK-826 06 Bratislava, Slovakia.

Phone: +421.2 .48234610$
The main aim of the treatment is to prevent invasive recurrence; however, understanding the biological behaviour of DCIS as well as prediction of prognosis is strongly inadequate. Local recurrence occurs in 5-30\% patients after breast conserving therapy, half of these recurrences are represented by invasive carcinoma (1). Responsible markers stratifying DCIS according to the recurrence risk are missing.

Numerous trials aim to identify new markers, which could help in selection of DCIS tumors with worse prognosis requiring an intensive treatment. Recent studies concerning DCIS recurrence and invasive progression noticed a possible prognostic significance of certain combination of biomarkers, of which importance alone is not clearly understood or marked as poor, such as COX-2, Ki67 and p16. These have shown to be associated with an invasive recurrence after conservative surgical treatment of 
DCIS when overexpression occurred in combination of all three markers. One of the most important trials in the field is the trial by Kerlikowske et al $(2,3)$.

The results of the Kerlikowske's trial showed a significantly higher invasive recurrence rate in subgroup of primary DCIS tumors with COX-2+Ki67+p16+ phenotype, no association was noticed between an invasive recurrence and age or tumor grade. A significant association was noticed between p16+COX-2-Ki67+ tumors and pure DCIS recurrence. The results of various trials showed shared characteristics between COX-2+p16+Ki67+DCIS and basal-like IDC tumors (with worse prognosis) (2).

One of the most consistent models of carcinogenesis hypothesizes gradual non obligatory progression of abnormal changes in terminal ductolobular unit through different stages. One of these important stages between non invasive precursors and invasive breast carcinoma is DCIS. Even though the carcinogenesis seems to be more complex, continual model is supported also by recent discoveries such as shared genetic alterations between precursor lesions and invasive breast carcinoma or an escalating risk of invasive cancer with precursor lesions (3).

Based on these facts, we suggest an association between COX-2, p16 and Ki67 markers with different stages of breast cancer and their perspective use in the evaluation of early breast tumors behaviour.

We decided to compare the expression of mentioned markers (COX-2, p16, Ki67 individually and in combinations) in different stages of breast cancer containing in situ component - in pure DCIS, microinvasive carcinoma (DCIS with microinvasive focuse/s up to $1 \mathrm{~mm}$ ) and in extensive in situ component of IDC (EIC, means DCIS comprising $>25 \%$ of tumor). We also performed a correlative analysis between the markers themselves and the characteristics regarded as prognostic or risk factors, such as patient's age, tumor grade, tumor necrosis, age of menarche, parity.

\section{Materials and methods}

40 patients with diagnosis of DCIS, microinvasive ductal carcinoma and invasive ductal carcinoma with EIC treated at 2nd Department of Gynaecology and Obstetrics, University hospital of Bratislava, from year 2000 to 2010, were included in this study. Immunohistochemical staining of paraffin-embedded tissue slides was used to assess and compare the expression of COX-2, p16 and Ki67. Clinical and historic data regarding the premorbid period, the course of the treatment and postoperative period were gained via questionnaire sent to all patients.

The study was officially approved as a retrospective clinical trial by the Independent Ethics Committee of Medical faculty, Comenius University and University Hospital of Bratislava on 7.2.2012. All patients participating in the study signed a written consent acknowledging the aspects of the trial.

Three patients were excluded from the study since their tissue samples were inadequate for histological analysis. There were 15 patients in the group of pure DCIS, 11 patients in the group of microinvasive carcinoma (pT1mic) and 11 patients with IDC with EIC. Paraffin tissue blocks from each patient were found in cooperation with the Department of Pathology according to histological examination after surgery. Four new series slides were made for each patient. One of the slides was stained with standard haematoxylin-eosin staining, acting as a background for diagnostic orientation. Slides were newly reviewed by pathologist in order to confirm the diagnosis and assess the analytic adequacy.

Patients' documentation review and primary postoperative pathologic results were used as source of information regarding diagnostic and surgical management, patient's age at the time of diagnosis and tumor characteristics (grade, size, comedo-necroses, multicentricity). Additionally, a questionnaire was sent to each patient in order to obtain history data such as parity, age of menarche, breast feeding, previous benign or malignant breast diseases, any previous malignancy, family history, use of hormonal contraception, previous irradiation of the chest and postoperative course of the disease.

\section{Immunohistochemical analysis}

Three paraffin-embedded slides from each patient were stained by monoclonal antibodies in order to identify biomarker expression in in situ component of each sample. For COX-2 identification, rabbit monoclonal antibody COX-2 (SP21) (Cell Marque) was used, CINtec Histology Kit (REF 9511) was used for p16 examination and rabbit monoclonal primary antibody Confirm Anti-KI-67 (Ventana) for evaluation of Ki67 expression. Staining was conducted according to corresponding protocol stated by antibody producers. COX-2 staining was regarded positive in case of cytoplasmatic staining with possibility of occasional membranous reaction. Besides the heterogeneity of COX-2 staining, we were aware of possible false COX-2 positivity in stained necrotic comedo-structures of DCIS or positive reaction in inflammatory stromal cells as well as intraluminal phagocytes. P16 immunopositive reaction was predominantly cytoplasmatic with possible nuclear reaction. Ki67 represents nuclear antigen, phenomenon of false positive reaction in proliferative lymphocytes was occasionally found similarly to COX-2 staining.

Two investigators evaluated COX-2, p16 and Ki67 stains according to the following protocols. In microinvasive carcinoma and IDC with EIC, marker expression was scored only in DCIS compound, evaluation was conducted under a standard magnification (200x).

For COX-2 evaluation, condensed Allred score was used similarly to Kerlikowske's trial to evaluate cytoplasmatic expression and both number of stained cells (expressed as Proportion score - PS 0-4) as well as intensity of the reaction (Intensity score - IS $0-3)$ were assessed. Allred combined score was expressed as a combination of PS and IS (0-8) and definite score was stated as follows: $0=$ Allred $0 ; 1=$ Allred 2, 3, 4; $2=$ Allred 5, 6 and $3=$ Allred 7, 8. Figure 1 shows a different intensity of COX-2 reaction.

Scoring of p16 was evaluated according to number of immunopositive cells without any regard to intensity of staining on the scale of $0-3(0=$ no reactive cell, $1=<25 \%$ positive cells, $2=25-75 \%$ cells, $3=75-100 \%$ ). Different grades of p16 stain reactions shows Figure 2. 

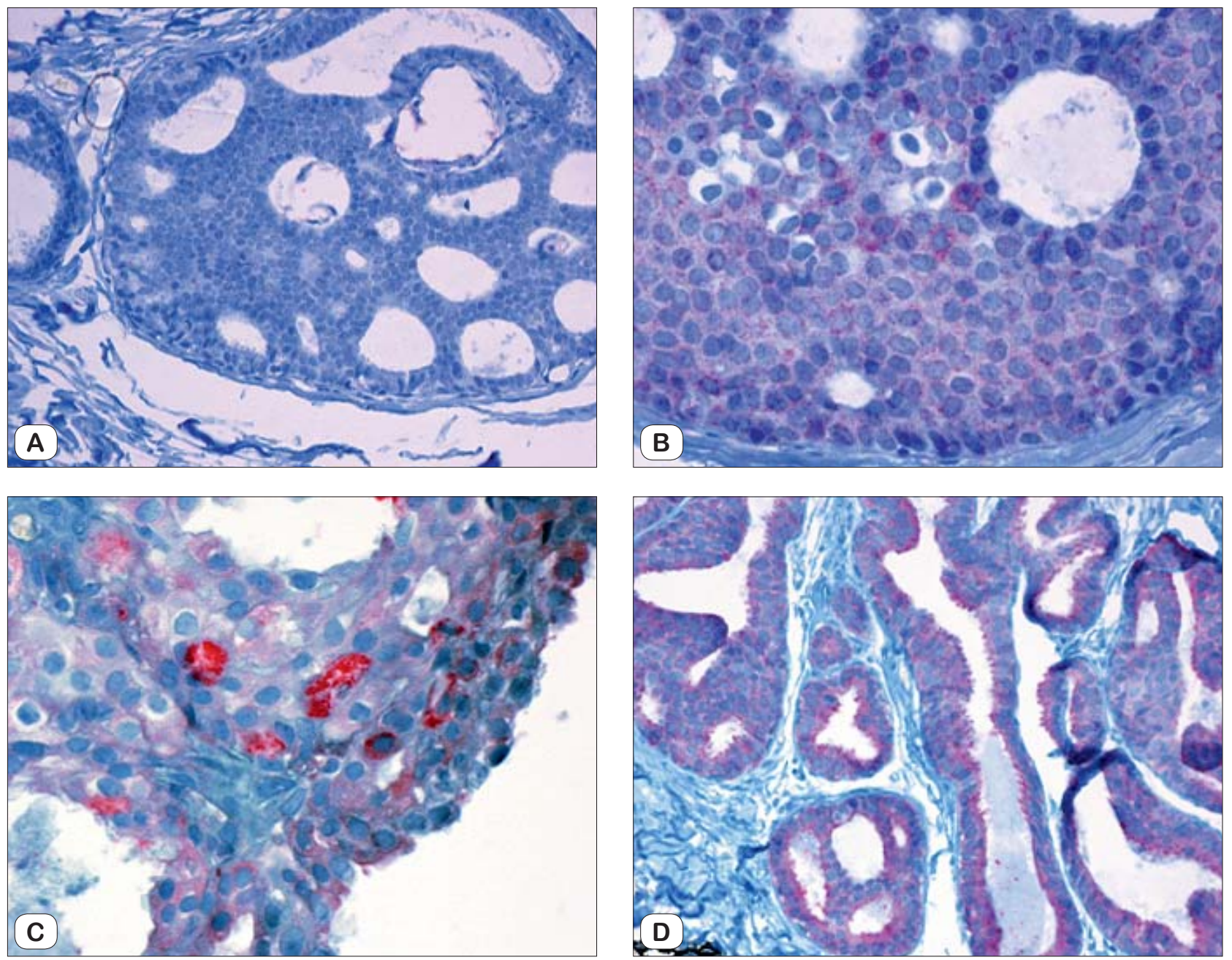

Fig. 1. COX-2 immunohisochemical staining, different intensity of reaction (Ondriaš, 2013). A) Negative reaction (intensity score 0), magnification x200, B) Low intensity of reaction (intensity score 1), magnification x200, C) Intermediate intensity of reaction (intensity score 2), magnification $x 400, D)$ Strong intensity of reaction, (intensity score 3), magnification $x 400$.

Ki67 staining was similarly evaluated according to the proportion of stained cells with score 0 for no immunoreactivity in the field, 1 for monocellular positivity, 2 for $1-10 \%$ positive cells and 3 for more than $10 \%$ of positive cells. Figure 3 presents particular grades of Ki67 reaction.

Tumors were regarded to overexpress COX-2 and p16 when scored with at least 2, Ki67 overexpression was stated in case of $>10 \%$ positive cells (score 3 ).

\section{Statistical analysis}

Statistical analysis was carried out using the SPSS 19.0 statistical software. For each analysed data, an appropriate statistical test according to the results of normality was used.

In single marker analyses, we examined the distribution of individual markers such as COX-2, p16 and Ki67 in three analysed groups (expression scores and overexpression incidence in DCIS, T1mic, IDC with EIC) as well as their association with characteristics previously described to be of prognostic significance such as age, grade of tumor and presence of comedo-necroses. We ex- amined the association between the markers themselves (relation COX-2-p16, COX-2-Ki67, p16-Ki67 in all patients). We performed multivariable analyses of combinations of two examined biomarkers and their distribution in the study subgroups.

To compared the variables in two samples using a non parametric two-sided Mann-Whitney test or parametric two-sided Student test in case of normal distribution. In more than two samples, non parametric Kruskal-Wallis analysis of variance or one way variance analysis (ANOVA) as parametric test was used.

All tests were done using the significance level $\alpha=0.05$.

\section{Results}

Among 37 patients in the cohort, 15 patients were diagnosed with DCIS according to histopathologic analyses (40.5\%), 11 with microinvasive carcinoma (T1mic, $29.7 \%$ ) and 11 with IDC and EIC $(29.7 \%)$. The mean age of the patients in all study groups was 52.73 (minimum 24, maximum 78, median 52) with most patients in the interval 60-65 years under normal distribution of 

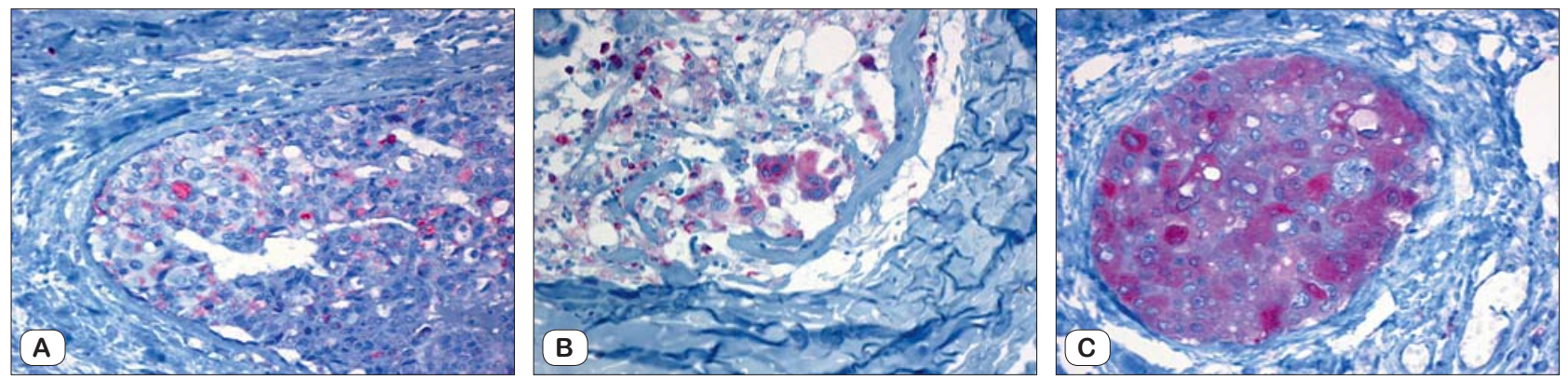

Fig. 2. p16 immunohistochemical staining, different intensity of reaction (Ondriaš, 2013). A) Low intensity of reaction, score 1, magnification x200, B) Intermediate intensity of reaction, score 2, magnification x200, C) High intensity of reaction, score 3, magnification x400.
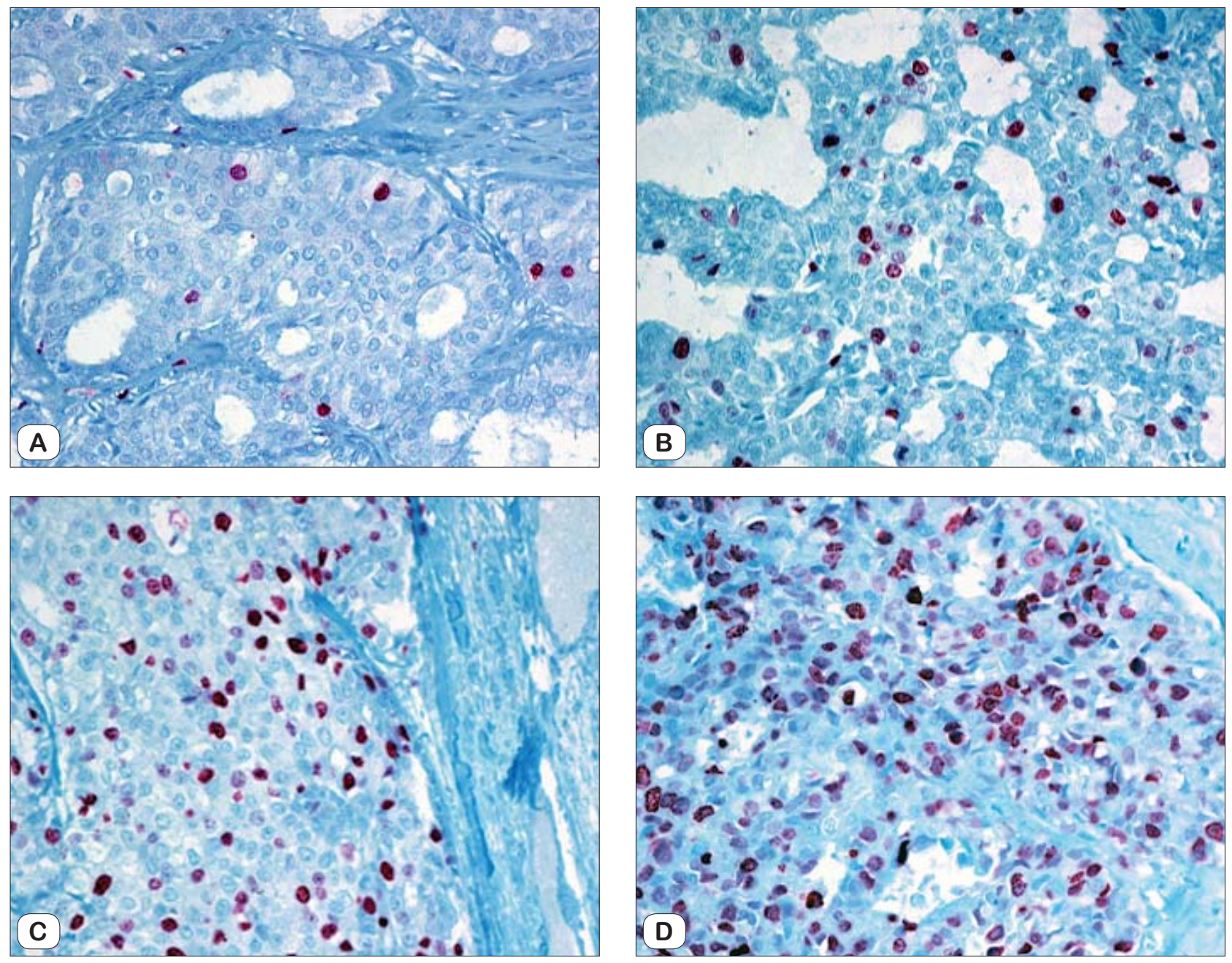

Fig. 3. Ki67 staining, different expression score (Ondriaš, 2013). A) Expression score 1 (monocelular reaction), magnification x200, B) Expression score 2, magnification x200, C) Expression score 3, magnification x200.

age in the studied groups. There was no significant difference in age distribution between the groups.

Questionnaires with historic data were received from 25 patients ( 8 from the group of DCIS, 10 from T1mic group and 7 from IDC+EIC group). In the whole cohort, the median age of menarche was 13.72 (minimum 12, maximum 16), subgroups did not sig- nificantly differ in this data distribution. However, all patients in pure DCIS group mentioned menarche at $>13$ years, in the group of microinvasive carcinoma and IDC was higher proportion of menarche age $<13$ years ( $60 \%$ in T1mic, $57.1 \%$ in IDC). In the analysis of parity, the mean number of deliveries was 2.48 for all patients (minimum 0, maximum 5). Concerning age at the time of 


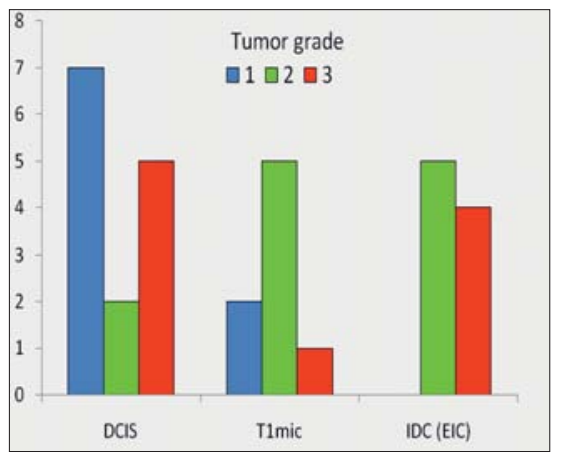

Fig. 4. Distribution of tumor grade among di- Fig. 5. COX-2 overexpression. agnoses DCIS, T1mic and IDC.

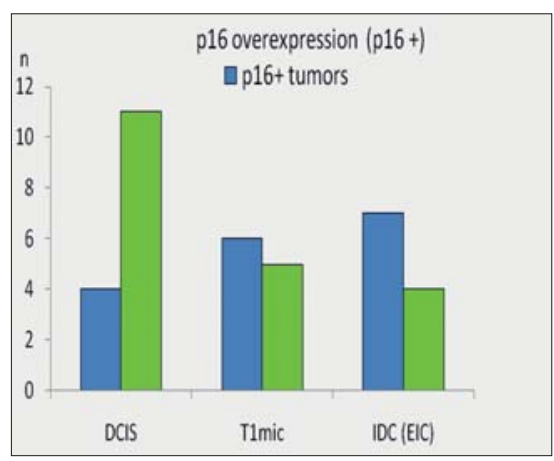

Fig. 7. p16 overexpression.

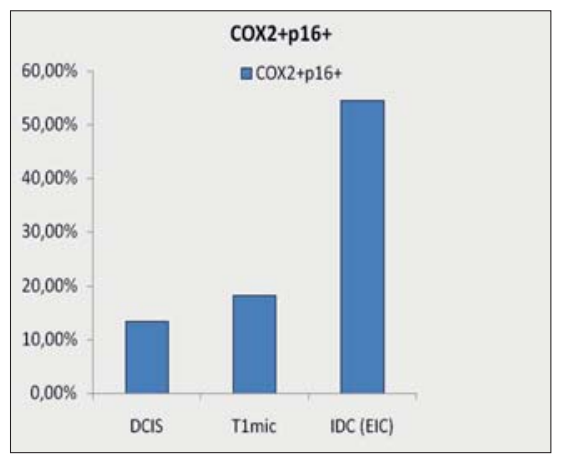

Fig. 8. Distribution of COX2+ and p16+ overexpression in combination (in \% within groups).

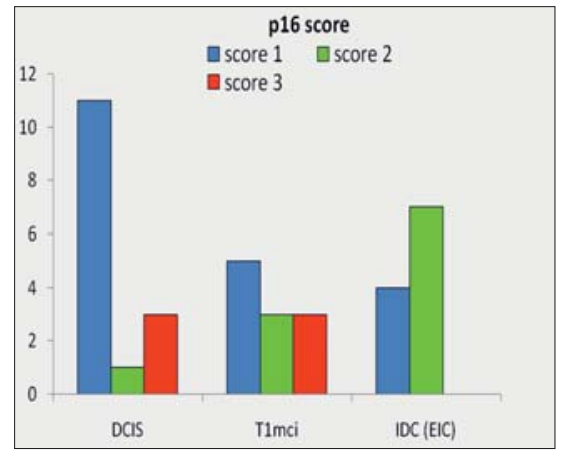

Fig. 6. Distribution of p16 score.

In the analysis of single marker expression among the subgroups, no statistically significant difference was found in distribution of COX-2 score and COX-2 overexpression (score 2, 3) nor in Ki67. However, a higher number of COX-2 overexpressing tumors was found in IDC group in comparison to the other groups (Fig. 5). The expression of the markers was evaluated exclusively in in situ component of the tumors (in case of IDC, expression was examined only in extensive intraductal component).

There was a significant difference in p16 score distribution with a higher number the 1 st delivery, we found out the mean age of 22.38 (minimum 17, maximum 30). The mean length of breast feeding was 6.45 months (minimum 2, maximum 18 months). $79.17 \%$ patients did not ever use hormonal contraception, $20.83 \%$ patients reported use of pills without length specification. There was no significant difference in these data distribution among the subgroups, nor in reported positive family history (breast carcinoma, other carcinoma) or previous benign breast disease. No patient reported previous irradiation of chest area. Group characteristics regarding historic data shows the Table 1.

The study groups did not significantly differ in the incidence of comedo- necrosis. There was a significant difference in the tumor grade distribution with a higher prevalence of low grade DCIS in the pure DCIS group (53.0\%) in comparison to T1 mic (25\%) and there was no low grade DCIS within EIC of DIC ( $0 \%)$ tumors. There was a higher number of high grade tumors within IDC (44.4\%) than in DCIS (35.7 \%); however, this was not significant (Fig. 4).

Tab. 1. Group description - mean value of characteristic data.

\begin{tabular}{lccccc}
\hline & Age & Parity Menarche & $\begin{array}{c}\text { Age at 1st } \\
\text { delivery }\end{array}$ & $\begin{array}{c}\text { Breast feeding in } \\
\text { months }\end{array}$ \\
\hline DCIS & 56,27 & 2,75 & 8 & 21,78 & 7 \\
\hline T1mic & 49,45 & 1,7 & 10 & 23,28 & 6,7 \\
\hline IDC (EIC) & 51,18 & 1,85 & 7 & 22,16 & 6,6 \\
\hline p & 0,353 & 0,436 & 0,122 & 0,356 & 0,848 \\
\hline
\end{tabular}

$\overline{\text { DCIS }}=$ ductal carcinoma in situ, T1mic $=$ micorinvasive carcinoma, IDC with EIC $=$ invasive ductal carcinoma with extensive intraductal component, $p=p$ value in statistical analysis of score 1 in the pure DCIS group (11 cases out of $15,73.3 \%$ ) in comparison to T1mic (5 out of 11 cases, $45.5 \%$ ) and EIC within DIC (4 from 11 cases, $36.4 \%$ ). The highest score 2 proportion was vice versa in the DIC group (7/11, 63.6\%), $27.3 \%$ (3 from 11 cases) was in T1mic and low proportion was in pure DCIS (1 out

Tab. 2. Biomarker overexpression.

\begin{tabular}{llccc}
\hline Diagnosis & & $\begin{array}{c}\text { COX-2 } \\
\text { overexpression }\end{array}$ & $\begin{array}{c}\text { P16 } \\
\text { overexpression }\end{array}$ & $\begin{array}{c}\text { Ki67 } \\
\text { overexpression }\end{array}$ \\
\hline DCIS & Count & 6 & 4 & 4 \\
& \%Within dg. & 40 & 26,7 & 26,7 \\
\hline T1mic & Count & 2 & 6 & 5 \\
& \%Within dg. & 18,2 & 54,5 & 45,5 \\
\hline IDC(EIC) & Count & 7 & 7 & 2 \\
& \%Within dg. & 63,6 & 63,6 & 18,2 \\
\hline
\end{tabular}

$\overline{\text { DCIS }}=$ ductal carcinoma in situ, $\mathrm{T} 1 \mathrm{mic}=$ micorinvasive carcinoma, IDC with $\mathrm{EIC}=$ invasive ductal carcinoma with extensive intraductal component, $\mathrm{p}=\mathrm{p}$ value in statistical analysis, dg. $=$ diagnosis

Tab. 3. Distribution of biomarker overexpression combinations.

\begin{tabular}{llccc}
\hline Diagnosis & & COX-2 + p16+ & COX-2+Ki67+ & p16+Ki67+ \\
\hline DCIS & Count & 2 & 2 & 2 \\
& \%Within dg. & 13,3 & 13,3 & 13,3 \\
\hline T1mic & Count & 2 & 0 & 18,2 \\
& \%Within dg. & 18,2 & & \\
\hline IDC (EIC) Count & 6 & 1 & 1 \\
& \%Within dg. & 54,5 & 9,1 & 9,1 \\
\hline
\end{tabular}

$\overline{\mathrm{DCIS}}=$ ductal carcinoma in situ, $\mathrm{T} 1 \mathrm{mic}=$ micorinvasive carcinoma, $\mathrm{IDC}$ with $\mathrm{EIC}=$ invasive ductal carcinoma with extensive intraductal component, $\mathrm{p}=\mathrm{p}$ value in statistical analysis, dg.= diagnosis 
from 15 patients, $6.7 \%$ ) (Fig. 6). There was no difference in the score 3 distribution. In the definite p16 overexpression analysis, there was no significant difference between the groups, however, we determined an obviously increasing tendency of p16 overexpression prevalence in the groups with invasive compound. 26.7 $\%$ in situ lesions (4 from 15 cases) overexpressed p16 in DCIS group, $54.5 \%(6 / 11)$ in T1mic group and $63.6 \%(7 / 11)$ in EIC within IDC) (Fig. 7).

In the marker-combination analysis, we found a gradually increasing proportion of COX-2 and 16 overexpressing tumors (COX$2+\mathrm{p} 16+)$ with severity of diagnosis $(13.3 \%, 2 / 15 \mathrm{COX}-2+\mathrm{p} 16+$ tumors in DCIS, $18.2 \%$ (2 from 11$)$ in T1mic, $54.5 \%$ (6 from 11$)$ in IDC with EIC) (Fig. 8), though this was not statistically significant. Distribution of other combinations did not differ between the groups.

Within the marker correlation analysis, there was a significant difference found by comparing the patients with COX-2 and p16 overexpression. Positive correlation was determined in the manner of a higher presence of p16 overexpression among COX-2 overexpressing tumors.

In the correlative analysis between the single biomarkers and prognostic characteristics of the tumors, no statistically significant difference was found between COX-2 or p16 expression and tumor grade, presence of necrosis or multicentricity. There was a significantly higher number of multicentric tumors in lesions overexpressing Ki67 in comparison to those without Ki67 overexpression (12\% of multicentric tumors among Ki67- lesions, 50 $\%$ among Ki67+ tumors) (Tabs 2 and 3).

\section{Discussion}

Our results showed a different expression pattern of p16 marker and COX2 in combination with p16 in DCIS, microinvasive carcinoma and invasive carcinoma (in its intraductal component) and the expression seemed to increase with the severity of the diagnosis.

P16 was the only single marker of which the score distribution correlated significantly with the rising severity of the tumors (the highest prevalence of score 1 in pure DCIS, the lowest in EIC within DIC), vice-versa the highest score 2 in EIC within DIC, lowest in pure DCIS). Even though there was no statistical significance in the score 3 distribution, in the definitive p16 overexpression analysis (overexpression $=$ score 2,3 ) the relation was maintained (the highest number of p16 overexpressing tumors was in DIC groups, the lowest in DCIS), though this was not significant. We think, this might be due to small number of group members. In Kerlikowske trial (2), p16 was the only individual marker correlating with an invasive recurrence after DCIS.

Further analyses showed a positive correlation between $\mathrm{p} 16$ and COX-2 overexpression. In the multivariate analysis, there was a higher proportion of COX-2+p16+ tumors in DIC than in T1mic and than in DCIS. Though in Kerlikowske trial Ki67 marker was associated with an invasive recurrence when in combination with COX2 and p16, the results from our study did not confirm a significant relation of Ki67 with an increasing severity of breast cancer diagnosis. In tumor characteristics analysis, tumor grade was associated with severity of diagnosis corresponding to previously known data.
A systematic review of studies concerning biological markers in DCIS and risk of breast recurrence was recently published in order to identify candidate markers or their combination associated with an increased risk of ipsilateral DCIS recurrence or subsequent invasive tumors (3). Of the 623 reviewed studies, the study by Kerlikowske et al was marked as one of the largest and arguably one of the most important in the field (3) as, in comparison to other studies, it was conducted on a group of patients who all underwent identical therapy. This retrospective case-control study showed a specific combination of COX-2, p16 and Ki67 markers significantly associated with invasive recurrences after DCIS. Eight-year risk of subsequent invasive cancer was statistically significantly higher in the group with initial DCIS lesions which were COX-2, p16 and Ki67 triple positive (COX-2+p16+Ki67+), risk of DCIS recurrence was significantly higher for initial DCIS with ER-ERBB2+Ki67+ or p16+COX2-Ki67+ phenotype. In the univariate analysis, p16 was the only marker significantly associated with an invasive recurrence (2).

The importance of the combination is reinforced also by other studies, that showed a common phenotype characteristics between COX-2+p16+Ki67+ tumors and basal like invasive tumors $(5,6)$.

Based on the continual model of carcinogenesis and mentioned results we hypothesized, that if combination of the COX-2, p16 and $\mathrm{Ki} 67$ is prognostic for DCIS future behaviour, expression levels of the markers could correlate also with different stages of breast carcinomas such as DCIS, microinvasive cancer and invasive cancer.

We examined the expression of mentioned markers within three groups of tumors compounding tumor compounding in situ component - in pure DCIS, microinvasive carcinoma (pT1mic) and EIC in invasive carcinoma in order to elucidate possible association with an increasing severity of breast lesions throughout carcinogenesis.

We see several strengths of our study. We did not evaluate biomarkers in a group of DCIS recurrences (DCIS or invasive) in order to avoid possible bias arising from factors influencing recurrence (different treatment, margin status etc.). It is the first study comparing the expression of biomarkers COX2, p16 and Ki67 in pure DCIS and in situ lesions in proximity to invasive compounds of various extant.

We are aware of several study limitations. It is still arguable, if DCIS within EIC is an entity independent from pure DCIS and if invasivity comes from site of DCIS, which may complicate reproducibility of our results. However, we presume this fact as molecular studies show a similar molecular phenotype of DCIS and coexisting invasive carcinoma (7) or same grade of DCIS within invasive carcinoma of the same grade (8). Also, the immunohistochemical interpretation of staining might be challenging due to certain level of subjectivity within the given evaluation scale as well as due to heterogeneity within the DCIS tumors. Probably the most significant limitation comes from the small cohort of patients in the particular groups. Due to the relatively small number of group members, we provided statistical analysis with combinations of two instead of three biomarkers (in comparison to study by Kerlikowske).

COX2, p16 and Ki67 is newly discovered combination of biomarkers with a potential prognostic value for DCIS recurrence risk. 
Our results showing different $\mathrm{p} 16$ and COX-2 phenotype of in situ lesions exisiting within different breast tumors indicate relation of the markers to the surrounding tissue, which may determine the biological behaviour of in situ lesions in future. The future research in greater cohorts could clarify this association and relation of 16 and COX-2 with progression of in situ lesions.

\section{References}

1. Tavassoli FA. Ductal intraepithelial neoplasia of the breast. Virchows Arch 2001; 438: 221-227.

2. Kerlikowske K, Molinaro AM, Gauthier ML, Berman HK, Waldman F, Bennington J, Sanchez H, Jimenez C, Stewart K, Chew K, Ljung B, Tlsty TD. Biomarker Expression and Risk of Subsequent Tumors After Initial Ductal Carcinoma In Situ Diagnosis. J National Cancer Inst 2010; 102: 627-637.

3. Lari SA, Kuerer HM. Biological markers in DCIS and risk of breast recurrence: A systematic review. J Cancer 2011; 2: 232-261.

4. Allred DC, Wu Y, Mao S et al. Ductal Carcinoma In situ and the Emergence of Diversity during breast cancer evolution. Clin Cancer Res 2008; 14: 370-378.

5. Boland GP, Butt IS, Prasad R, Knox WF, Bundred NJ. COX-2 expression is associated with an aggressive phenotype in ductal carcinoma in situ. Brit J Cancer 2004; 90: 423-429.

6. Gauthier ML, Berman HK, Miller C et al. Abrogated stress response distinguishes basal like tumors and DCIS lesions associated wit subsequent tumor events. Cancer Cell 2007; 12 (5): 479-491.

7. Gupta S, Deka L, Gupta R, Pant L, Singh S. Molecular phenotypes of ductal carcinoma-in-situ and invasive ductal carcinoma: a comparative study. Indian J Pathol Microbiol 2012; 55 (1): 43-46.

8. Subhawong A, Subhawong T, Nassar T et al. Most Basal-like Breast Carcinomas Demonstrate the Same Rb À/p16+ Immunophenotype as the HPV-related Poorly Differentiated Squamous Cell Carcinomas Which They Resemble Morphologically. Am J Surg Pathol 2009; 33: 163-175.

9. Raspollini M, Taddei GL. Cocylooxygenase-2: A Novel Target in Human Solid Tumors. Curr Oncol Rep 2007; 9: 96-101.

10. Peurala E, Koivunen P, Haapasaari KM, Bloigu R, Jukkola-Vuorinen $A$. The prognostic significance and value of cyclin D1, CDK 4 and p16 in human breast cancer. Breast Cancer Research 2013; 15: R5.

11. Dowsett M, Nielsen TO, A’Hern R et al. Assessment of Ki67 in Breast Cancer: Recommendations from the International Ki67 in Breast Cancer Working Group. J Natl Cancer Inst 2011; 103: 1656-1664.

12. Urruticoechea A, Smith IE, Dowsett M. Proliferation Marker Ki-67 in Early Breast Cancer. J Clin Oncol 2005; 23: 7212-7220.

13. Divvela AKCh, Challa SR, Tagaram IK. Pathogenic role of cyclooxygenase-2 in cancer. J Health Sci 2010; 56 (5): 502-516.

14. Subhawong AP,Subhawong T, Nassar H, Kouprina N, Begum S, Vang R, Westra WH, Argani P. Most Basal-like Breast Carcinomas Demonstrate the Same Rb /p16+ Immunophenotype as the HPV-related Poorly Differentiated Squamous Cell Carcinomas Which They Resemble. Am J Surg Pathol 2009; 33 (2).
15. Bohn OL, Fuertes-Camilo M, Navarro L, Saldivar J, SanchezSosa S. p16INK4a expression in basal-like breast carcinoma. Int J Clin Exp Pathol 2010; 3: 600-607.

16. Herschkowitz JI, He X, Fan C, Perou CM. The functional loss of the retinoblastoma tumor suppressor is a common event in basal-like and luminal B breast carcinomas. Breast Cancer Res 2008; 10: R75.

17. Denkert C, Winzer K-J, Muller B-M, Weichert W, Pest S, Kobel M, Kristiansen G, Reles A, Siegert A, Guski H, Hauptmann S. Elevated expression of cyclooxygenase-2 is a negative prognostic factor for disease survival and overall survival in patients with breast cancer. Cancer 2003; 97: 2978-2987.

18. Kerlikowske K, Molinaro AChaI, Ljung BM et al. Characteristics associated with recurrence among women with ductal carcinoma in situ treated by lumpectomy. J Natl Cancer Inst 2003; 95 (22): 1692-1702.

19. Ringberg A, Nordgren H, Thorstensson $S$ et al. Histopathological risk factors for ipsilateral breast events after breast conserving treatment for ductal carcinoma in situ of the breast - results from the Swedish randomised trial. Eur J Cancer 2007; 43 (2): 291-298.

20. Ringberg A, Anagnostaki L, Anderson H, Idvall I, Fernö M. Cell biological factors in ductal carcinoma in situ (DCIS) of the breast-relationship to ipsilateral local recurrence and histopathological characteristics. Eur J Cancer 2001; 37 (12): 1514-1522.

21. Cornfield DB, Palazzo JP, Schwartz GF et al. The prognostic significance of multiple morphologic features and biologic markers in ductal carcinoma in situ of the breast: a study of a large cohort of patients treated with surgery alone. Cancer 2004; 100 (11): 2317-2327.

22. Barnes N, Haywood P, Flint P, Knox WF, Bundred NJ. Survivin expression in in situ and invasive breast cancer relates to COX-2 expression and DCIS recurrence. Br J Cancer 2006; 94 (2): 253-258.

23. Tamimi RM, Baer HJ, Marotti $\mathbf{J}$ et al. Comparison of molecular phenotypes of ductal carcinoma in situ and invasive breast cancer. Breast Cancer Res 2008, 10: R67.

24. Allred DC, Harvey JM, Berardo M, Clark GM. Prognostic and predictive factors in breast cancer by immunohistochemical analysis. Mod Pathol 1998; 11 (2): 155-168.

25. Bryan BB, Schnitt SJ, Collins LC. Ductal carcinoma in situ with basal-like phenotype: a possible precursor to invasive basal-like breast cancer. Mod Pathol 2006; 19 (5): 617-621.

26. Livasy CA, Perou CM, Karaca G. Identification of a basal-like subtype of breast ductal carcinoma in situ. Hum Pathol 2007; 38 (2): 197-204.

27. Crawford YG, Gauthier ML, Joubel A, Mantei K, Kozakiewicz K, Afshari CA, Tlsty TD. Histologically normal human mammary epithelia with silenced $\mathrm{p} 16$ (INK4a) overexpress COX-2, promoting a premalignant program. Cancer Cell 2004; 5 (3): 263-273.

28. Hua M, Peluffoa G, Chend H, Gelmand R, Schnittb S, Polyaka K. Role of COX-2 in epithelial-stromal cell interactions and progression of ductal carcinoma in situ of the breast. Proc Natl Acad Sci USA 2009; 106 (9): 3372-3377.

29. Ernster VL, Ballard-Barbash R, Barlow WE et al. Detection of ductal carcinoma in situ in women undergoing screening mammography. J Natl Cancer Inst 2002; 94 (20): 1546-1554. 